\title{
Exploring Interaction Space as Abstraction Mechanism for Task-Based User Interface Design
}

\author{
Christian M. Nielsen \\ NNIT \\ Brennum Park \\ DK-3400 Hillerød \\ Denmark \\ cmne@nnit.com
}

\author{
Michael Overgaard \\ KMD \\ Selma Lagerlöfsvej 300 \\ DK-9220 Aalborg East \\ Denmark \\ mio@kmd.dk
}

\author{
Michael B. Pedersen \\ ETI \\ Bouet Møllevej 3-5 \\ DK-9400 Nørresundby \\ Denmark
}

mbpedersen@gmail.com

\author{
Jan Stage \\ Aalborg University \\ Department of Computer Science \\ Fredrik Bajers Vej 7 \\ DK-9220 Aalborg East, Denmark \\ jans@cs.aau.dk
}

\author{
Sigge Stenild \\ Aalborg University \\ Department of Computer Science \\ Fredrik Bajers Vej 7 \\ DK-9220 Aalborg East, Denmark \\ ZilentNinja@gmail.com
}

\begin{abstract}
Designing a user interface is often a complex undertaking. Modelbased user interface design is an approach where models and mappings between them form the basis for creating and specifying the design of a user interface. Such models usually include descriptions of the tasks of the prospective user, but there is considerable variation in the other models that are employed. This paper explores the extent to which the notion of interaction space is useful as an abstraction mechanism to reduce the complexity of creating and specifying a user interface design. We present how we designed a specific user interface through use of design techniques and models that employ the notion of interaction space. This design effort departed from the task models in an object-oriented model of the users' problem and application domains. The lessons learned emphasize that the notion of interactions spaces is a useful abstraction mechanism that can help user interface designers exploit object-oriented analysis results and reduce the complexity of designing a user interface.
\end{abstract}

\section{Categories and Subject Descriptors}

H.5.2 [Information Interfaces and Presentation]: User Interfaces - Graphical user interfaces (GUI), Theory and methods. D.2.2 [Software Engineering]: Design Tools and Techniques - User interfaces, Object-oriented design methods.

\section{General Terms}

Design, Experimentation, Human Factors, Languages.

Permission to make digital or hard copies of all or part of this work for personal or classroom use is granted without fee provided that copies are not made or distributed for profit or commercial advantage and that copies bear this notice and the full citation on the first page. To copy otherwise, or republish, to post on servers or to redistribute to lists, requires prior specific permission and/or a fee.

Conference'06, Month 1-2, 2006, City, State, Country.

Copyright 2006 ACM 1-58113-000-0/00/0004 ...\$5.00.

\section{Keywords}

User interface design, Abstraction, Object-oriented, Domain models.

\section{INTRODUCTION}

Models and specifications are key elements in many software development projects. It has been documented that software development based on specifications produce systems with a high level of functionality and robustness [1]. Accordingly, it has been suggested that specifications are crucial in projects where high technical complexity makes software reliability and verification paramount, e.g. batch systems, process control systems, complex systems and safety-critical systems [18]. In many interactive systems, the user interface accounts for a large part of the code and is a major source of complexity [1].

In software engineering, it is generally agreed that developers use abstraction and decomposition to cope with complexity in software design [6][9][22][24]. This use of abstraction and decomposition as vehicles for human reflection has also been denoted as the analytical mode of operation [13]. The analytical mode of operation relies on abstraction mechanisms, which are concepts or language constructs that support abstraction and decomposition. The notion of objects and classes is an example of abstraction mechanisms that support abstraction and decomposition of phenomena in a problem domain [5][21]. Designers employ these concepts for taming the complexity of a software design effort. In user interface design, the notion of task is an example of an abstraction mechanism that is used in several model notations.

More recently, the importance of abstraction in user interface design has been emphasized for other reasons. The broad variety of mobile devices that are being developed are proving to be a challenge for user interface designers because they offer only limited and different means of interaction and small screens [6][8]. Moreover, the diversity of the mobile platform makes it imperative to design on an abstract level [3]. This latter argument can be generalized to any type of multi-platform system. 
Model-based user interface design aims to support an analytical mode of operation in user interface design with a range of models and mappings between them [10]. It has been emphasized that within this approach, there is no consensus about the models to use and the notations employed in the models [3].

The purpose of this paper is to move beyond specific model notations by exploring an abstraction mechanism for user interface design. The aim is to identify an abstraction mechanism and establish to what extent it supports abstraction and decomposition in user interface design. In the following section 2 , we present related work in model-based user interface design and discuss potential abstraction mechanisms. The results presente din this paper are based on a case study of the process of designing the user interface of an interactive location-aware mobile system to support communication in a specific safety-critical application domain. The domain was the fuel department of a coal-based power plant. This case study is presented in section 3 . Section 4 provides an overview of the results from an object-oriented analysis that was conducted prior to the case study. Section 5 presents the process and result of designing the user interface of the mobile application. Section 6 elaborates on the lessons learned from the case study. Finally, section 7 provides the conclusion.

\section{RELATED WORK}

Model-based user interface design includes two major streams of work. The first stream focuses on model features and mappings between models. The aim is to develop computerized tools to support the models and transformations between them, e.g. [4][20]. A key topic in this stream is denoted as the mapping problem, which refers to the need for handling transformations from one model to another [3][10]. The main problem in this stream is to derive models of concrete user interfaces from more abstract models. This has been described as the abstract-toconcrete gap [15]. Some tools handle this gap by requiring human intervention in certain activities of the design process [23].

The second stream of work in model-based user interface design focuses on the creative process that is conducted by human designers. The aim is to provide tools and techniques that support humans in handling the complexities involved in the design task. This has also been denoted as design heuristics [19].

Our work is within the second stream. With this approach it is crucial to develop strong abstraction mechanisms that can help designers tame the complexities of the user interface design task.

The literature reflects three different types of abstraction mechanisms that have been suggested for user interface design. The first type originates from user interface technology. In the Cameleon reference framework [2], the most abstract description of a user interface is a logical model that is based on elements that are abstractions of existing widgets [10]. Thus this approach employs an abstraction mechanism that is derived from existing user interface technology.

The second type of abstraction mechanisms originates from the domain of the prospective users of the system. Some focus on the tasks of the users. Tasks are a key element of the application domain of a system [12]. Most task-driven design techniques exploit hierarchically organized task structures with temporal dependencies between sub-tasks to derive models of user interfaces [20]. Others focus on the objects of the users' work.
This is a key element in the problem domain of a system [12]. Finally, there are attempts to include both tasks and objects [11].

The notion of interaction space is an interesting abstraction mechanism for model-based design of user interfaces. It is used in the Wisdom (Whitewater Interactive System Development with Object Models) method [16][17]. An interaction space is a part of the user interface that facilitates some specific interaction between the user and the system. It is a class that will be used to generate elements for the user interface. Examples of interaction spaces are higher-level widgets such as windows, menus and panels. It has a graphical representation that can be used in combination with models based on the UML notation.

The concept of views has been suggested as a vehicle for design of the user interface. For a given task, the designer defines how many views are needed to support that task. Then the sub-tasks of that task are distributed on the views [23]. This idea is similar to interaction spaces in the sense that an abstract concept forms the basis for design. However, a view represents a screen or part of a screen and thereby, it is less abstract than an interaction space that can represent any mean for interaction..

In the rest of this paper, we explore to what extent the notion of interaction space is useful as an abstraction mechanism for user interface design. The discussion is based on a case study that is presented in the following section.

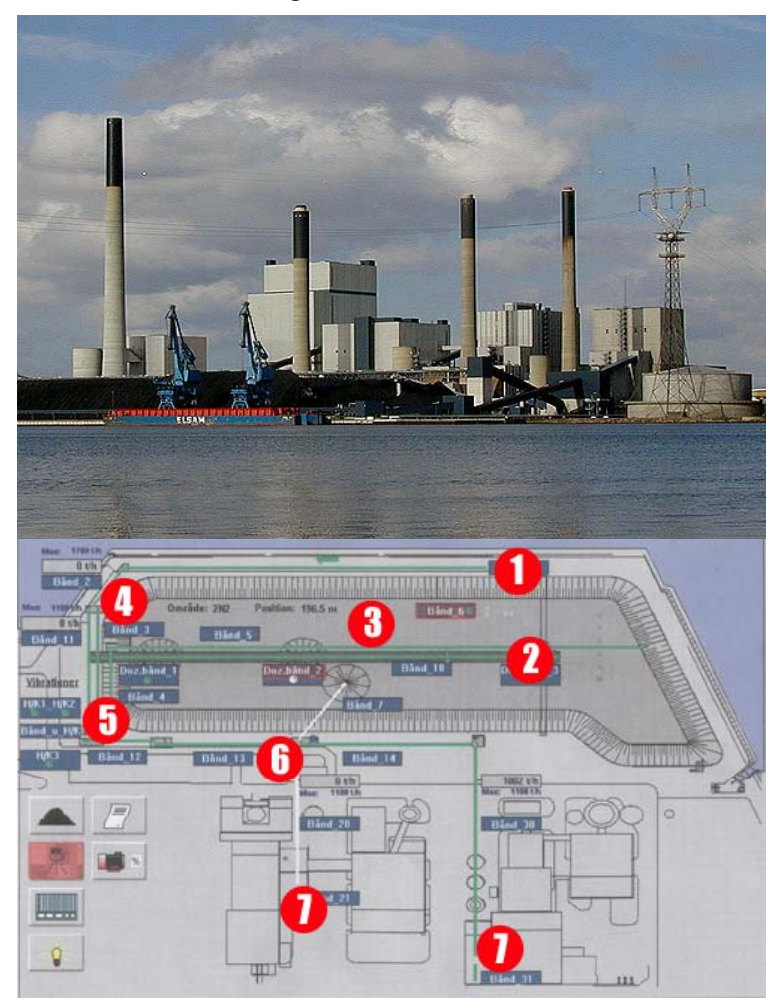

Figure 1. Nordjyllandsværket and the fuel department

\section{CASE STUDY}

The case study was conducted at Nordjyllandsværket, a coalbased power plant situated in Northern Jutland in Denmark, see Figure 1 . The plant produces central heating, electricity, and byproducts that are used in the production of cement. We limited the 
case study to the fuel department of the power plant, see the diagram in Figure 1.

The plant is divided into two independent production plants (the two locations \#7). The coal for the two plants is supplied from a central storage area (locations \#2 and \#3). The fuel-department is responsible for delivering the coal used in the two production plants, amounting daily to 5000 tons of coal for each.

The employees in the fuel department continuously monitor and control the transportation of coal. They must ensure that the correct amount of coal arrives to the correct location and that the coal has certain properties and quality. To ensure this, the coal is processed (locations \#4 and \#5) before transporting it to the final location (location \#7). Another important task is to prevent the coal in the storage area from self-combusting.

The employees perform a variety of different tasks to ensure that the needed amount of coal is delivered to the two production plants. To coordinate the tasks described above, quick and easy communication is important, and in some cases even essential in order to carry out the job in a safe and efficient manner. At the present, the devices used for communicating are VHF-radios (walkie-talkies), DECT wireless phones, and some times mobile phones. The control tower (location \#6) is the only location where all the necessary information is accessible and it is also the place where employees can operate and control most of the machinery.

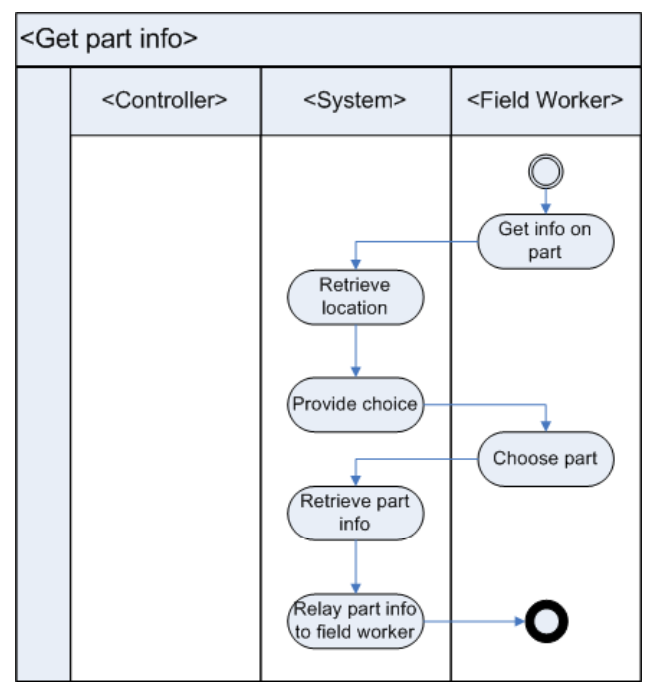

Figure 2. Essential task flow diagram for getting information about a part of a machine

Every element of the transportation of coal can be controlled through the existing system, but only through a complex coordination process. When a problem arises, which cannot be solved from the control room, for example in the Grinder building (location \#5), the person situated here trying to solve a problem does not have access to the relevant information. Furthermore specific parts of the machinery can only be controlled from inside the Grinder building. The only way to gain access to the information systems in the control room is by communicating with a person in the control room either by phone or walkietalkie.
Often the phones are not usable because of the weak signal; hence the only tool for communicating is the walkie-talkie. Several problems are related to the use of this device. Many conveyorbelts run underground, which disrupts the signal, and the machines and conveyor belts are often placed inside concrete buildings, which also disrupts the signal. Finally, there is a deafening noise inside these buildings, which makes talking to each other difficult, and using any kind of mobile device for verbal communication is virtually impossible.

\section{ANALYSIS RESULTS}

Prior to the case study, we had conducted an object-oriented analysis of a location-aware mobile communicator to support employees in the application domain presented above. This was based on OOA\&D, a typical object-oriented analysis method [12]. Below, we present the relevant results from this analysis.

The overall idea of the application was summarized in the following system definition:

- Functionality: Communication device with machine state indication and support for communication

- Application Domain: Transport of coal around the power plant, preparation and mixing of coal, monitoring of conveyer belts and problem solving/prevention in production line

- Conditions: Safety critical, noisy environment, dusty conditions, above- and underground, employees have basic IT training/knowledge

- Technology: Pocket PC with Microsoft visual studio 2003 .Net and WLAN

- Objects: Employee, mobile unit, conveyer belt, magnet, screener, grinder, control room computer

- Responsibility: Context-aware mobile communication support system that monitors production line state and facilitates cooperation and communication in a noisy environment

Two main models were produced during analysis. The users' work tasks in the application domain were described in terms of use cases. We identified nine use cases that should be supported by the system. We modelled each of these. There are two roles in the problem domain: controller and field worker. In order to model their different responsibilities and to emphasize the purpose of the system, we expressed the work in terms of activity models with responsibilities. Wisdom [17] denotes this as an essential task flow diagram. For an example, see Figure 2.

The problem domain was described by a class diagram and a set of statechart diagrams. The class diagram depicts the physical locations, the communication, and the users relevant to our system, see Figure 3. For each class, there was also a state chart diagram that describes the detailed behaviour for objects from this class.

Finally, the analysis produced a list of 22 functions that the system should include. The list describes each function and specifies its complexity and type. 


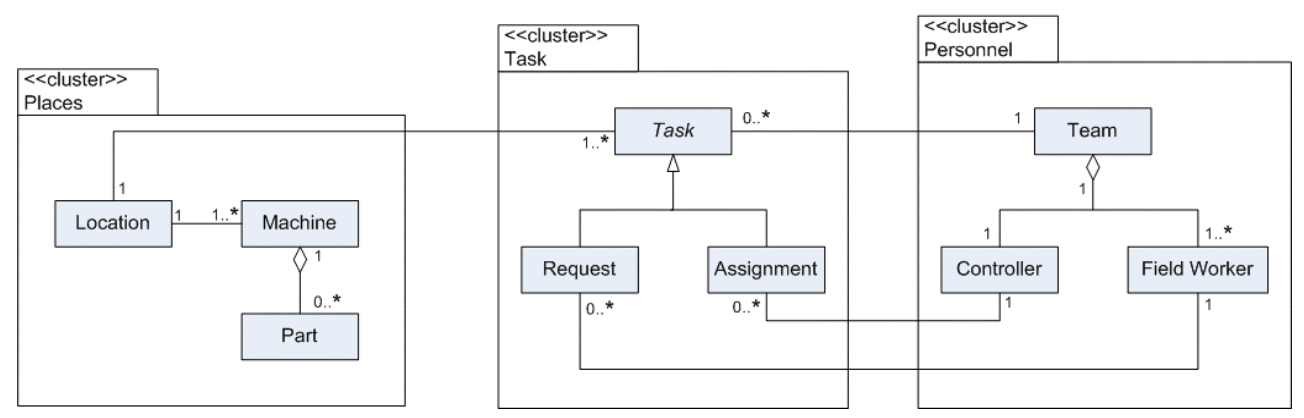

Figure 3. Class diagram for the whole problem domain

\section{USER INTERFACE DESIGN}

The user interface design departed directly from the results of the object-oriented analysis.

\subsection{Individual Interaction Models}

We started making individual interaction models. For each essential task flow diagram, we made an individual interaction model. This model provides a set of interaction spaces that are needed to support a user that is carrying out the task.

An individual interaction model is defined by taking the corresponding task flow diagram and on the right hand side of this diagram drawing up the interaction spaces that is needed to support each activity in the diagram.

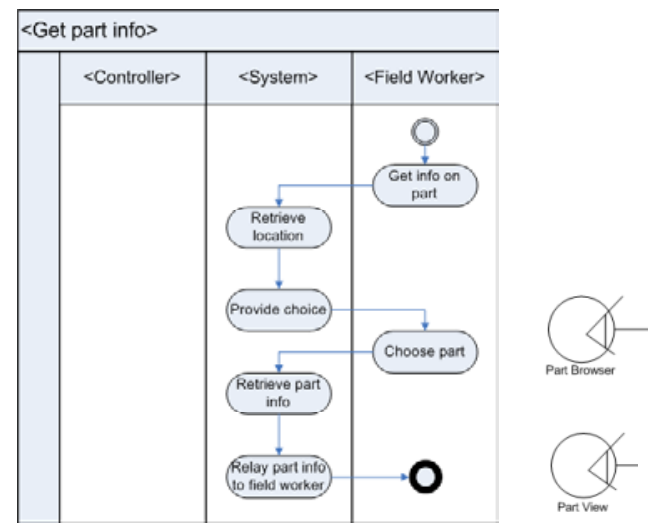

Figure 4. Essential task flow diagram for getting information about a part of a machine with interaction spaces added

This is illustrated in Figure 4, where we have added two interaction spaces on the right hand side. These two are examples of two interaction spaces that can be applied in many cases: browser and view [17]. The browser is an interaction space that shows and enables selection of a number of objects of a class. In this case it shows the parts that are visible at a specific location, and the user is able to select one of these objects. The view provides information about a single object. In this case it is information about the part the user has chosen.

The next step is to add what Wisdom refers to as task classes that are related to individual interaction spaces. However, here we discovered a source of confusion. The whole diagram describes how a task is carried out, and as part of that, we describe something that is also referred to as tasks. In order to differentiate, we denoted the activity related to an interaction space as an interaction task.

Thus for each interaction space in the diagram, we describe the interaction task or tasks that are supported by that interaction space. In Figure 5 we have added interaction tasks for the two interaction spaces.

This is done for each essential task flow diagram. In our case, we had nine diagrams, so we ended up with 9 individual models like the one shown in Figure 5.

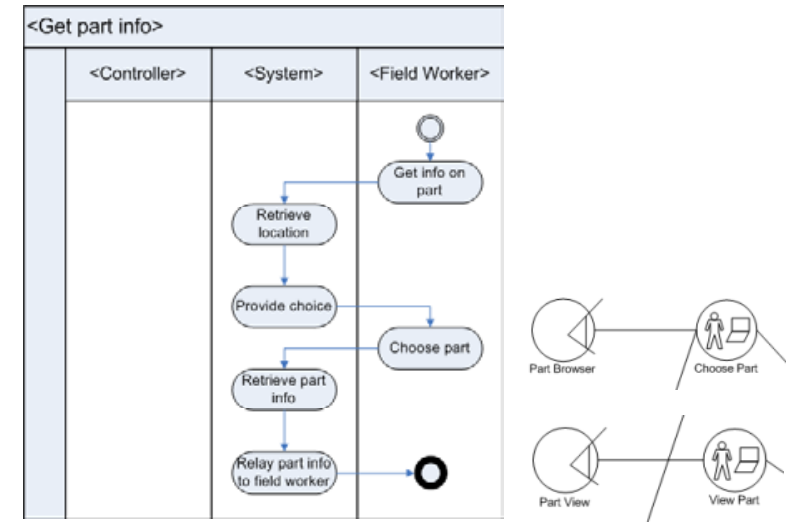

Figure 5. Essential task flow diagram for getting information about a part of a machine with interaction spaces and interaction tasks added

\subsection{General Interaction Model}

When all of the individual interaction models are complete, they are combined into a general interaction model. The general interaction model describes all the interaction spaces and interaction tasks that the system must support.

In our case, we had two roles, controller and field worker, and in the task models, we had divided the description between them. Thus we were able to relate the interaction spaces to each role. Therefore, we decided to make a separate general interaction model for each of the roles. Figure 6 shows the general interaction model for the Field worker. A general interaction model was also made for the Controller This is illustrated in 
Figure 7. For both models, the first version of the general interaction model only included the two leftmost columns.

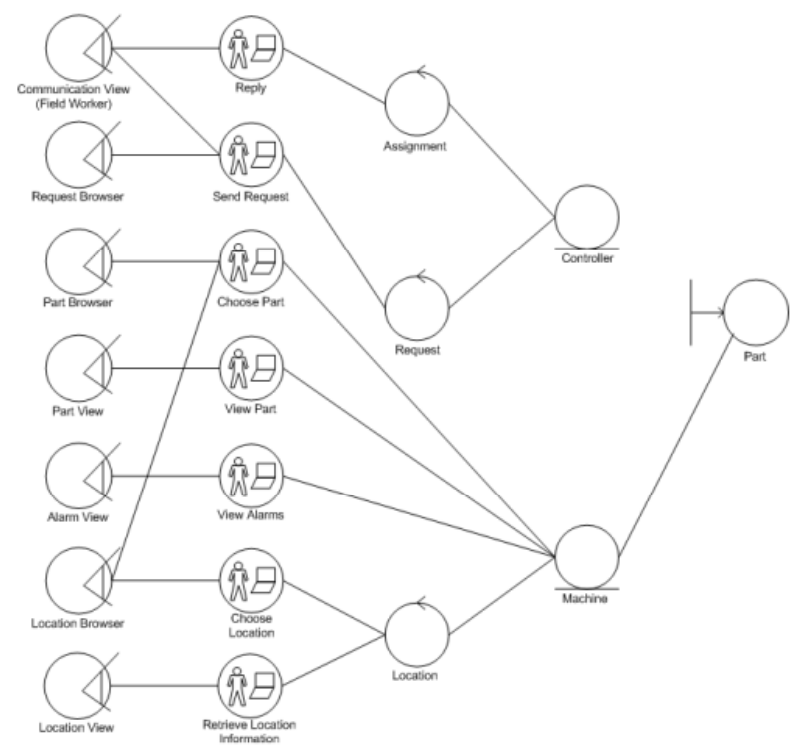

Figure 6. The general interaction model for the Field worker

In the process of mapping the individual interaction models into the general interaction model, repeated task classes and interaction spaces were eliminated.

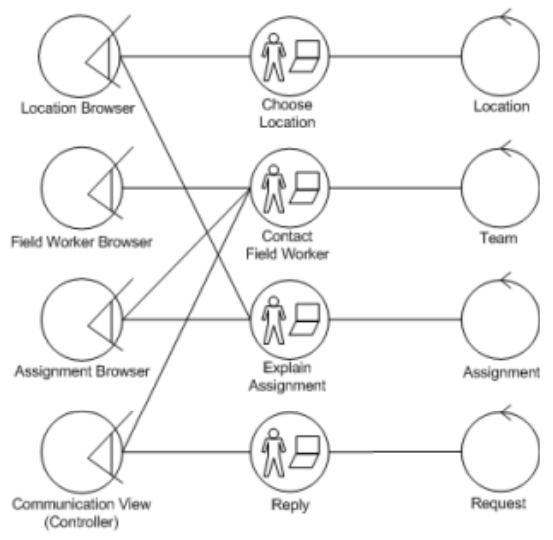

Figure 7. The general interaction model for the Controller

\subsection{Relation to Problem Domain Classes}

Up to this point, the user interface design involved only the task models from the results of the object-oriented analysis. However, we also used the class diagram, see

Figure 3. This was done by relating each interaction task to the objects it involved. This is illustrated in the rightmost columns of Figure 6. For example, the choose part interaction task involves an object from the Machine class which in turn involves an object from the Part class.

The different symbols used for the problem domain classes are not important for this example. Thus this part of the general interaction diagram describes the relation between the interaction model and the problem domain model that is described on the overall level by the class diagram.
For the Controller, we also described the relation between the interaction tasks and the classes in the class diagram. This is the rightmost column in Figure 7.

Both of these general interaction models illustrate the relation between interaction spaces and problem domain classes. Each interaction task is related to one problem domain class. This is a useful criterion for assessing the interaction tasks you have defined; if an interaction involves more than one class, it might be considered to break it down further.

\subsection{Updating Task Flow Diagrams}

The work process described in a task flow diagram involves a number of interaction tasks. For the diagram in Figure 5, there are two interaction tasks: choose part and view part. We have used this relationship to describe more structure in the task flow diagrams. We did this by creating an overall state for each interaction task. This is illustrated in Figure 8.

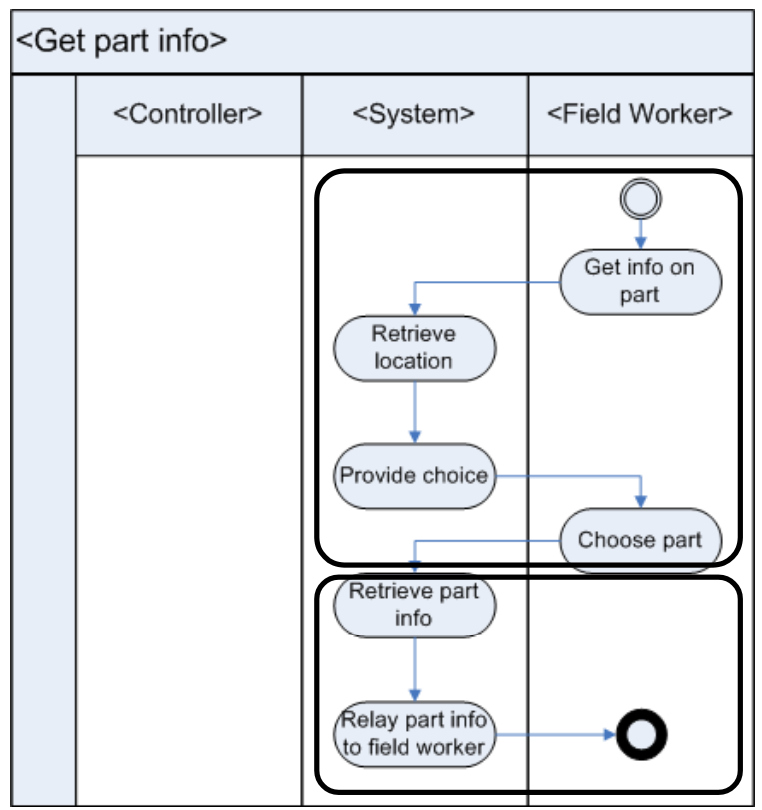

Figure 8. Essential task flow diagram for getting information about a part of a machine with two overall states for interaction tasks added

This provides a clear relation between the use of the interaction spaces of the system and the description of the users' work in the task flow diagrams. It describes the sequence in the use of the system. In Wisdom, this is modelled in a so-called dialogue model [17].

\subsection{Creating the Presentation Model}

The general interaction diagrams were used for creating a presentation model for the system. The presentation model describes the classes that will be used to generate object in the user interface. It is a single model for the whole system that is based on the interaction spaces. The interaction spaces are transformed to classes one by one, and they are related to each other with object-oriented structures. 


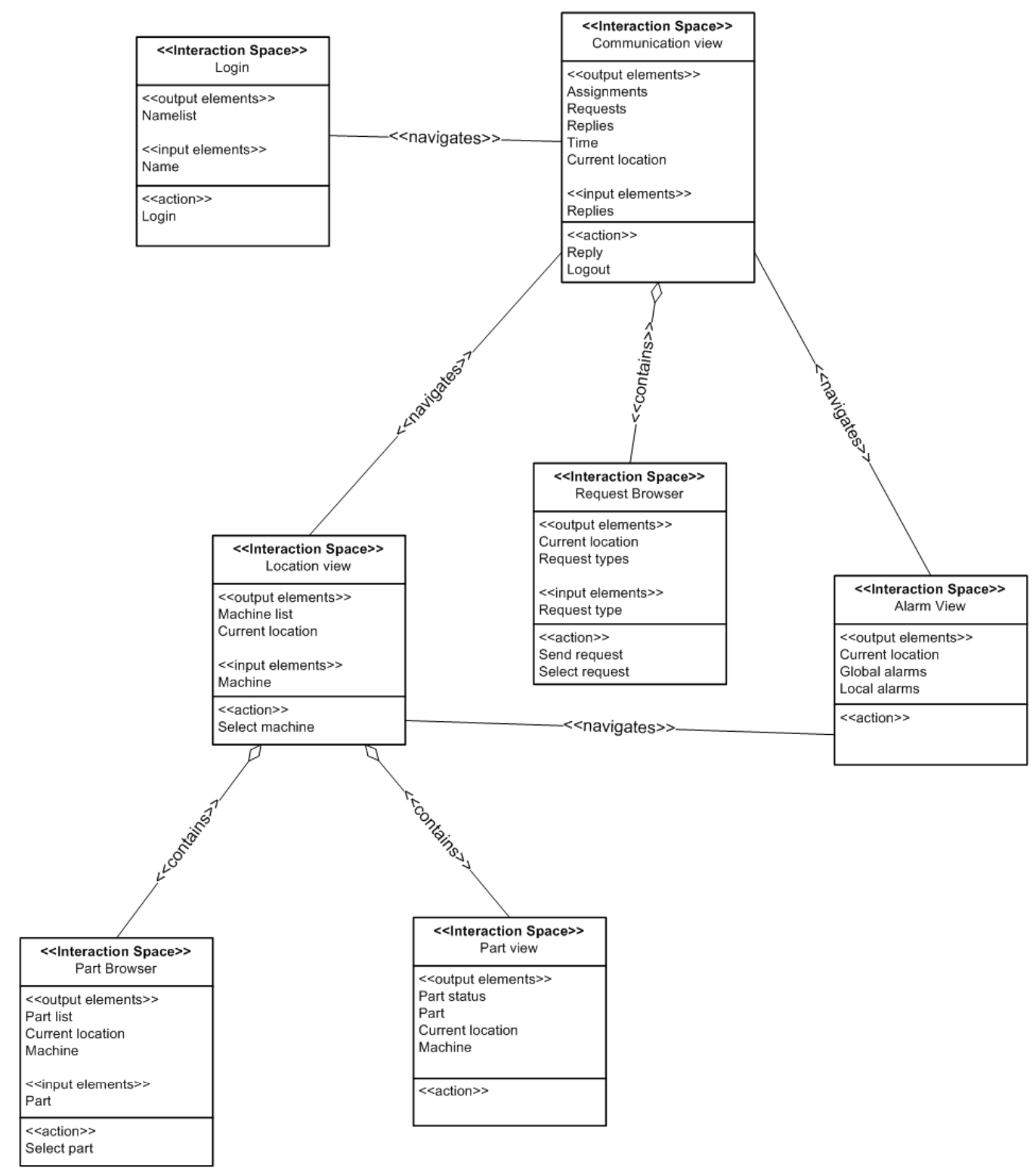

Figure 9. The presentation model for the whole system

The presentation model for the whole system is shown in

Figure 9. The attributes of the classes in the diagram are defined from the detailed class diagram for the problem domain, and the operations are defined by distributing the function list from analysis on the individual presentation model classes.

\subsection{Designing the User Interface}

The last activity was to design the concrete user interface from the presentation model and the task flow diagrams. This encompassed two concurrent flows of activities, corresponding to the dialogue model and the task flow diagrams. The presentation model describes the elements that are included in a specific window, and the relevant task flow diagram describes the order in which they are employed by the user when solving a task.

When the concrete user interface was designed, it turned out to be difficult to group objects on screens, and it was crucial to take the screen size and the different means for interaction into account.

The design of one screen in the system is illustrated in Figure 10. This window is the one that support the task described in Figure 2. It includes three classes Location View, Part View and Part Browser in the presentation model, see the lower left part of Figure 9. 
Based on this design, we implemented a functional prototype. A window of this prototype is shown in Figure 10.

The prototype was implemented in the .Net Compact Framework. This forced some alterations to the design of the individual screens of the prototype. One major change was that the tab panes had to be moved to the bottom of the screen, as it was impossible to place them at the top. Also using the navigation button of the PDA for navigation between the tab panes had to be excluded due to an error in the .Net Compact Framework. Instead, navigation was accomplished by finger touch. Furthermore, it was impossible to determine the size of each tab pane, which made it questionable whether they where large enough to be used for finger touch navigation. Finally, the bottom of the screen could not be used as it was reserved for other PDA functionality.

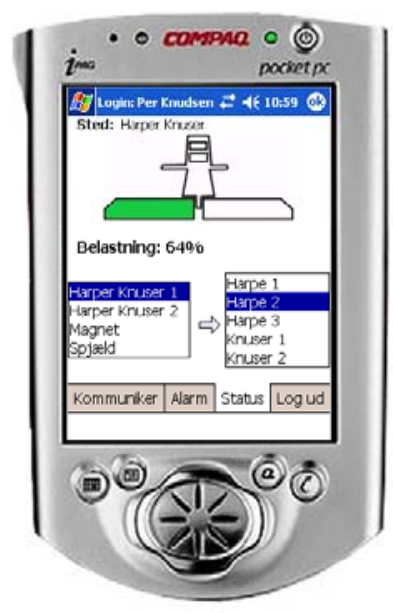

Figure 10. One screen of the user interface of the communication device

The usability of the implemented prototype was evaluated in two ways: (1) a heuristic inspection performed by five usability experts [14], and (2) a field evaluation in cooperation with five employees at the power plant, who are the prospective users of the mobile system. In the field test, the workers used the system while members of the development team controlled the communication with the PDA in order to simulate a realistic work process. After the work tasks were completed, the user was interviewed about the usability of the system.

The overall results of these evaluations showed us that the system was indeed usable. The employees were positive towards actually using the system instead of the walkie-talkie. One of them even said that he actually found this way of communicating better because he was sometimes disturbed by all the non-work related talk taking place over the VHF-radio. The employees all agreed that, especially in the locations, where the noise level is high, the system would be very useful, and they would want to use the system, if it was fully implemented. Despite disagreeing on how detailed information that the system should provide in the status and alarm sections, they all found the presented information useful in both solving problems and monitoring the production line.

\section{LESSONS LEARNED}

Through the case study of designing the user interface for the mobile communication device for the power plant, we learned the following lessons:

- The results from the object-oriented analysis was a productive basis for user interface design

- The models that we used were well related to each other

- The notion of interaction spaces was a useful abstraction mechanism (we got hold of the task)

- The abstraction allowed us to focus on the essential elements and defer detailed design issues

- The design process enabled us to produce a novel user interface design

- Methodological support for the detailed user interface design was needed

The design process departed from the results of an objectoriented analysis. Selected results from the analysis were directly applicable, and we had no need to make additional analysis activities. Thus the case study demonstrates that it is possible to tie object-orientation and user interface design together. The results from the application domain analysis provided the overall framework, as the task models were used to derive and specify interaction spaces. The problem domain model was used to qualify the description of interaction spaces and to provide details for the design of interface elements.

We used a combination of models from an object-oriented analysis method [12] and Wisdom [17]. This combination worked well in the sense that it covered the necessary activities of user interface design.

The notion of interaction spaces was used to define elements in the user interface that would support the users' individual tasks. This enabled us to handle the complexity of the user interface without getting burdened with details about widgets and other concrete elements. Thereby, the notion provided a useful abstraction mechanism.

The abstraction was linked to the models of the users' tasks. In our case study, we used essential task models, but other task models might be used instead. This relation meant that we could describe the essential elements of the user interface first. Later, we could deal with the detailed design issues, and the decisions on these details did not affect the decisions on the essential elements.

The user interface we designed grew out of the case we worked with. The idea of illustrating the machine and providing two connected menus with machines and part was new for this type of application. We have designed other communication devices for other application domains. However, this design was substantially different. The abstraction in terms of interaction spaces facilitated the creative process.

When we created the detailed design of the user interface, we had no methodological support. In this process it would have been useful to have guidelines for defining the specific details of the individual user interface elements.

We also discovered a fundamental problem in the design process. If our task flow were too detailed, it became very 
complicated to describe interaction spaces and interaction tasks. We solved this problem by relating interaction tasks to the problem domain model's class diagram. An interaction task should involve one class, and handle everything related to that class. Thus the class diagram defines the level of granularity for the interaction model.

\section{CONCLUSION}

This paper describes experience from a case study where we conducted a model-based design of the user interface of a mobile communicator for a safety-critical domain. In this process, we exploited results from an object-oriented analysis. The design process was supported by selected model notations from the Wisdom method. The case study indicated that the notion of interaction spaces is useful as an abstraction mechanism in the design process. It can help user interface designers exploit object-oriented analysis results and reduce the complexity of designing a user interface. The user interface design process was successful to the extent that the different activities produced results that were relevant for the following activities, we created a novel design and the usability evaluation of a functional prototype gave positive results.

These conclusions are based on a single case study. In addition, the case was to design a mobile communication system for a safety-critical domain. It would be interesting to try out the presented approach on other cases.

\section{ACKNOWLEDGMENTS}

We are grateful to the employees of the fuel department at Nordjyllandsværket for giving us the opportunity to use the fuel department as the case in our design process.

\section{REFERENCES}

[1] Boehm, B., Gray, T., and Seewaldt, T. Prototyping versus Specifying: A Multiproject Experiment. IEEE Trans. Software Eng. SE-10, 3 (May 1984), 290-303.

[2] Calvary, G., Coutaz, J., Thevenin, D., Limbourg, Q., Bouillon, L. and Vanderdonckt, J. A Unifying Reference Framework for Multi-Target User Interfaces. Interacting with Computer, 15, 3 (2003), 289-308.

[3] Clerckx, T., Luyten, K., and Coninx, K. The Mapping Problem Back and Forth: Customizing Dynamic Models while Perserving Consistency. In Proceedings of TAMODIA 2004, ACM, 33-42.

[4] Clerckx, T., Winters, F. and Coninx, K. Tool Support for Designing Context-Sensitive User Interfaces using a Model-Based Approach. In Proceedings of TAMODIA 2005, ACM, 11-18.

[5] Coad, P. and Yourdon, E. Object-Oriented Analysis, second edition. Prentice-Hall, Englewood Cliffs, New Jersey, 1991.

[6] Dey, A. K. and Abowd, G. D. Towards a Better Understanding of Context and Context-Awareness. In Proceedings of CHI 2000, ACM.

[7] Dijkstra, E. Notes on structured programming. In Structured Programming. Academic Press, London, 1972, $1-82$.
[8] Dunlop, M. D. and Brewster, S. A. The challenges of mobile devices for human computer interaction. Personal and Ubiquitous Computing, 6, 4 (2002).

[9] Langefors, B. Theoretical Analysis of Information Systems. Studentlitteratur, 1966.

[10] Limbourg, Q. and Vanderdonckt, J. Adressing the Mapping Problem in User Interface Design with UsiXML. In Proceedings of TAMODIA 2004, ACM, 155-163.

[11] Mahfoudi, A., Abed, M. and Abid, M. Towards a User Interface Generation Approach Based on Object Oriented Design and Task Model. In Proceedings of TAMODIA 2005, ACM, 135-142.

[12] Mathiassen, L., Munk-Madsen, A., Nielsen, P. A. and Stage, J. Object-Oriented Analysis \& Design. Marko Publishing, Aalborg, 2000.

[13] Mathiassen, L. and Stage, J. The Principle of Limited Reduction in Software Design. Information Technology \& People, 6, 2-3 (1992), 171-185.

[14] Nielsen, J. and Molich, R. Heuristic Evaluation of User Interfaces. In Proceedings of CHI 1990, ACM, 249-256.

[15] Nobrega, L. Nunes, N. J. and Coelho, H. DialogSketch: Dynamics of the Canonical Prototypes. In Proceedings of TAMODIA 2005, ACM, 19-25.

[16] Nunes, N. J. and Cunha, J. F. Wisdom: A Software Engineering Method for Small Software Development Companies. IEEE Software, 2001.

[17] Nunes, N. J. and Cunha, J. F. Wisdom - Whitewater Interactive System Development with Object Models. In M. van Harmelen (Ed.), Object Modeling and User Interface Design. Addison-Wesley, 2001.

[18] Parnas, D. Software Aspects of Strategic Defense Systems. Communications of the ACM, 28, 12 (Dec. 1985), 13261335.

[19] Prinebeau, C. and Vanderdonckt, J. Exploring Design Heuristics for User Interface Derivation from Task and Domain Models. In Proceedings of CADUI 2002, 103-110.

[20] Reichart, D. Forbrig, P. and Dittmar, A. Task Models as a Basis for Requirements Engineering and Software Execution. In Proceedings of TAMODIA 2004, ACM, 5158.

[21] Rumbaugh, J., Blaha, M., Premerlani, W., Eddy, S., and Lorensen W. Object-Oriented Modelling and Design. Prentice-Hall, Engelwood Cliffs, New Jersey, 1991.

[22] Wirth, N. Systematic Programming. An Introduction. Prentice-Hall, Englewood Cliffs, New Jersey, 1973.

[23] Wolff, A., Forbrig, P., Dittmar, A. and Reichart, D. Linking GUI Elements to Tasks - Supporting an Evolutionary Design Process. In Proceedings of TAMODIA 2005, ACM, 27-34.

[24] Wulf, W. Languages and structured programs. In R.T. Yeh (Ed.), Current Trends in Programming Methodology. Prentice-Hall, New Jersey, 1977. 